\title{
Classification of All Single Traveling Wave Solutions of Fractional Perturbed Gerdjikov-Ivanov Equation
}

\author{
Zhao Li $\mathbb{D}^{1,2}$ and Tianyong Han ${ }^{1,2}$ \\ ${ }^{1}$ College of Computer Science, Chengdu University, Chengdu 610106, China \\ ${ }^{2}$ Key Laboratory of Pattern Recognition and Intelligent Information Processing, \\ Institutions of Higher Education of Sichuan Province, Chengdu University, Chengdu 610106, China \\ Correspondence should be addressed to Zhao Li; lizhao10.26@163.com
}

Received 4 August 2021; Accepted 24 August 2021; Published 3 September 2021

Academic Editor: Mostafa M. A. Khater

Copyright (C) 2021 Zhao Li and Tianyong Han. This is an open access article distributed under the Creative Commons Attribution License, which permits unrestricted use, distribution, and reproduction in any medium, provided the original work is properly cited.

The fractional perturbed Gerdjikov-Ivanov (pGI) equation plays a momentous role in nonlinear fiber optics, especially in the application of photonic crystal fibers. Constructing traveling wave solutions to this equation is a very challenging task in physics and mathematics. In the current article, our main purpose is to give the classifications of traveling wave solutions of the fractional pGI equation. These results can help physicists to further explain the complex fractional pGI equation.

\section{Introduction}

The nonlinear Schrödinger equation (NLSE) is usually used to describe many problems in nonlinear optics, quantum mechanics, fluid dynamics, plasmas, and so on [1-5].
Among them, NLSE with cubic nonlinearity, namely, the perturbed Gerdjikov-Ivanov (pGI) equation, is the most studied [6-11], which is given by

$$
i \frac{\partial u}{\partial t}+a \frac{\partial^{2} u}{\partial x^{2}}+b|u|^{4} u=i\left[c u^{2} \frac{\partial \bar{u}}{\partial x}+\delta_{1} \frac{\partial u}{\partial x}+\delta_{2} \frac{\partial\left(|u|^{2} u\right)}{\partial x}+\theta \frac{\partial|u|^{2}}{\partial x} u\right]
$$

where $u(t, x)$ is a complex valued wave profile with respect to time and space variables. $\bar{u}$ is the complex conjugation of $u$. $c$ stands for the nonlinear dispersion coefficient. $\delta_{1}, \delta_{2}$, and $\theta$ are known parameters.
In recent years, with the development of fractional derivative, the fractional perturbed Gerdjikov-Ivanov equation has been widely concerned by many researchers in the following form:

$$
i D_{t}^{\alpha} u+a D_{x}^{2 \alpha} u+b|u|^{4} u=i\left[c u^{2} D_{x}^{\alpha} \bar{u}+\delta_{1} D_{x}^{\alpha} u+\delta_{2} D_{x}^{\alpha}\left(|u|^{2} u\right)+\theta D_{x}^{\alpha}|u|^{2} u\right], \quad 0<\alpha<1,
$$

where $u=u(t, x)$ represents unknown functions about time $t$ and space $x$, respectively. $\bar{u}$ is the complex conjugation of $u$.
$D_{t}^{\alpha} u$ and $D_{x}^{\alpha} u$ are the conformable fractional derivative [12-14]. 
In recent years, the traveling wave solution of the fractional pGI equation has attained a lot of interest in the filed of nonlinear science. Some explicit traveling wave solutions of the fractional pGI equation have been reported [15-17]. In Reference [15], Li and his collaborators studied the space-time fractional pGI equation by the fractional H-expansion method. In Reference [16], Ghanbari and Dumitru obtained the optical solutions of the fractional GI equation by using the generalized exponential rational function method. In Reference [17], Zulfiqar and Ahmad explored the optical solution of the fractional pGI equation by the Tanh method and Tanhcoth method, respectively. The main purpose of this paper is to give the classifications of traveling wave solutions of the fractional pGI equation by using the complete discrimination system method for polynomial, which is an important method to find the exact traveling wave solution of the fractional partial differential equation (FPDE). In general, the FPDE can be simplified to the following complete discriminant system by traveling wave transformation:

$$
\Psi^{\prime}(\xi)=F\left(\Psi, \phi_{1}, \ldots, \phi_{m}\right),
$$

where $\phi_{1}, \ldots, \phi_{m}$ are parameters. $F\left(\Psi, \phi_{1}, \ldots, \phi_{m}\right)$ is thirdorder polynomial, fourth-order polynomial, or fifth-order polynomial. Then, integrating equation (3) once, we obtain

$$
\xi-\xi_{0}=\int \frac{\mathrm{d} \Psi}{\sqrt{F\left(\Psi, \phi_{1}, \ldots, \phi_{m}\right)}}
$$

where $\xi_{0}$ is the integration constant. The readers can refer to $[2,4,18]$ for details of this method.

The structure of this paper is as follows. In Section 2, we simplify equation (2) to the nonlinear ordinary differential equation by fractional traveling wave transformation. Then, the complete discrimination system is used to construct the classification of all single traveling wave solutions of the fractional pGI equation. In Section 3, we give a summary.

\section{Traveling Wave Solutions of System (2)}

Consider the fractional traveling wave transformation as follows:

$$
\begin{aligned}
u(t, x) & =U(\xi) e^{i \eta}, \\
\xi & =\frac{x^{\alpha}}{\alpha}-v \frac{t^{\alpha}}{\alpha}, \\
\eta & =-k \frac{x^{\alpha}}{\alpha}+w \frac{t^{\alpha}}{\alpha},
\end{aligned}
$$

where $v$ is the phase component, $w$ represents the wavenumber, and $k$ is the frequency.

Substituting (5) into (2) and separating the real and imaginary part, we obtain

$$
a U^{\prime \prime}-\left(w+a k^{2}+\delta_{1} k\right) U+k\left(c-\delta_{2}\right) U^{3}+b U^{5}=0,
$$

and

$$
\begin{aligned}
& v=-\delta_{1}-2 a k, \\
& \theta=-\frac{1}{2}\left(c+3 \delta_{2}\right) .
\end{aligned}
$$

Multiplying equation (6) by $U^{\prime}$ and integrating it with respect to $\xi$, we obtain

$$
\frac{a}{2}\left(U^{\prime}\right)^{2}+\frac{b}{6} U^{6}+\frac{k\left(c-\delta_{2}\right)}{4} U^{4}-\frac{w+a k^{2}+k \delta_{1}}{2} U^{2}+d=0
$$

where $d$ is the integral constant. Next, we fix $U=\sqrt{\psi}$, then equation (8) can be converted into

$\left(\psi^{\prime}\right)^{2}=-\frac{4 b}{3 a} \psi^{4}-\frac{2 k\left(c-\delta_{2}\right)}{a} \psi^{3}+\frac{4\left(w+a k^{2}+k \delta_{1}\right)}{a} \psi^{2}-\frac{8 d}{a} \psi$.

(1) If $d=0$, equation (9) can be simplified as

$$
\left(\psi^{\prime}\right)^{2}=\psi^{2}\left(b_{3} \psi^{2}+b_{2} \psi+b_{1}\right)
$$

where $b_{3}=-(4 b / 3 a), \quad b_{2}=-\left(2 k\left(c-\delta_{2}\right) / a\right)$, and $b_{1}=\left(4\left(w+a k^{2}+k \delta_{1}\right) / a\right)$. Then, equation (10) can be written in the following integral form:

$$
\int \frac{\mathrm{d} \psi}{\psi \sqrt{b_{3} \psi^{3}+b_{2} \psi+b_{1}}}= \pm 2\left(\xi-\xi_{0}\right) \text {. }
$$

Denote $\Delta=b_{2}^{2}-4 b_{1} b_{3}$, where $\Delta$ is the discriminant of polynomial $b_{3} \psi^{3}+b_{2} \psi+b_{1}$. According to the complete discriminant method of second-order polynomials, the classification of all solutions of equation (11) has the following three cases:

Case 1.1. Suppose that $\Delta=0$. From equation (11), we can get

$$
\pm \frac{b_{2}}{b_{3}} \sqrt{\left|b_{3}\right|}\left(\xi-\xi_{0}\right)=\ln \left|\frac{\psi-\left(b_{2} / 2 b_{3}\right)}{\psi}\right| \text {. }
$$

Case 1.2. Suppose that $\Delta>0$. When $b_{3}>0$, we have from (11) 


$$
\begin{aligned}
& \pm \sqrt{b_{3}}\left(\xi-\xi_{0}\right)=\frac{1}{\sqrt{\gamma_{1} \gamma_{2}}} \ln \frac{\left[\sqrt{\left(-\gamma_{2}\right)\left(\psi-\gamma_{1}\right)}-\sqrt{\left(-\gamma_{1}\right)\left(\psi-\gamma_{2}\right)}\right]^{2}}{|\psi|}, \\
& \pm \sqrt{b_{3}}\left(\xi-\xi_{0}\right)=\frac{1}{\sqrt{\gamma_{1} \gamma_{2}}} \ln \frac{\left[\sqrt{\gamma_{2}\left(\psi-\gamma_{1}\right)}-\sqrt{\gamma_{1}\left(\psi-\gamma_{2}\right)}\right]^{2}}{|\psi|} \\
& \pm \sqrt{b_{3}}\left(\xi-\xi_{0}\right)=\frac{1}{\sqrt{-\gamma_{1} \gamma_{2}}} \arcsin \frac{\left(-\gamma_{2}\right)\left(\psi-\gamma_{1}\right)+\left(-\gamma_{1}\right)\left(\psi-\gamma_{2}\right)}{|\psi|\left|\gamma_{1}-\gamma_{2}\right|}
\end{aligned}
$$

When $b_{3}<0$, we have from (11)

$$
\begin{aligned}
& \pm \sqrt{-b_{3}}\left(\xi-\xi_{0}\right)=\frac{1}{\sqrt{-\gamma_{1} \gamma_{2}}} \ln \frac{\left[\sqrt{\left(-\gamma_{2}\right)\left(\psi+\gamma_{1}\right)}-\sqrt{\gamma_{1}\left(\psi-\gamma_{2}\right)}\right]^{2}}{|\psi|}, \\
& \pm \sqrt{-b_{3}}\left(\xi-\xi_{0}\right)=\frac{1}{\sqrt{-\gamma_{1} \gamma_{2}}} \ln \frac{\left[\sqrt{\gamma_{2}\left(-\psi+\gamma_{1}\right)}-\sqrt{\left(-\gamma_{1}\right)\left(\psi-\gamma_{2}\right)}\right]^{2}}{|\psi|}, \\
& \pm \sqrt{-b_{3}}\left(\xi-\xi_{0}\right)=\frac{1}{\sqrt{\gamma_{1} \gamma_{2}}} \arcsin \frac{\left[\sqrt{\left(-\gamma_{2}\right)\left(\psi+\gamma_{1}\right)}-\sqrt{\gamma_{1}\left(\psi-\gamma_{2}\right)}\right]^{2}}{|\psi|},
\end{aligned}
$$

where $\gamma_{1}=\left(-b_{2}+\sqrt{\Delta} / 2 b_{3}\right) \quad$ and $\quad \gamma_{2}=\left(-b_{2}-\right.$

Case 1.3. Suppose that $\Delta<0$. Then, we have $\left.\sqrt{\Delta} / 2 b_{3}\right)$.

$$
\pm \sqrt{b_{1}}\left(\xi-\xi_{0}\right)=\frac{1}{\sqrt{b_{1}^{2}}} \ln \left|\frac{-\left(1 / 2 b_{2} \sqrt{b_{1}}\right) \psi+\sqrt{b_{1}}-\sqrt{b_{3} \psi^{2}-b_{2} \psi+b_{1}}}{\psi}\right|, \quad\left(b_{1}>0\right)
$$

(2) If $d \neq 0$, equation (9) can be simplified as

$$
\left(\psi^{\prime}\right)^{2}=\psi\left[-\frac{4 b}{3 a} \psi^{3}-\frac{2 k\left(c-\delta_{2}\right)}{a} \psi^{2}+\frac{4\left(w+a k^{2}+k \delta_{1}\right)}{a} \psi-\frac{8 d}{a}\right] .
$$

Then, equation (16) can be written in the following integral form:

$$
\left\{\begin{array}{l}
\Delta=-27\left(\frac{2 p^{3}}{27}+r-\frac{q r}{3}\right)^{2}-4\left(q-\frac{p^{2}}{3}\right)^{3}, \\
D_{1}=q-\frac{p^{2}}{3},
\end{array}\right.
$$

$\int \frac{\mathrm{d} \psi}{\sqrt{\varepsilon \psi\left(\psi^{3}+p \psi^{2}+q \psi+r\right)}}= \pm 2 \sqrt{-\frac{b \varepsilon}{3 a}}\left(\xi-\xi_{0}\right)$,

where $\varepsilon= \pm 1, p=(3 b / k(c-\delta)), q=-\left(3\left(w+a k^{2}\right.\right.$ $\left.\left.+k \delta_{1}\right) / b\right)$, and $r=(6 d / b)$.

Denote where $\Delta$ is the discriminant of polynomial $G(\psi)=\psi^{3}+p \psi^{2}+q \psi+r$. According to the complete discriminant method of third-order polynomials, the classification of all solutions of equation (11) has the following four cases: 
Case 2.1. Assume that $\Delta=0, D_{1}<0$. Denote $G(\psi)=\left(\psi-\beta_{1}\right)^{2}\left(\psi-\beta_{2}\right)$, where $\beta_{1} \neq \beta_{2}$ and $\beta_{2}>0$.
If $\varepsilon=1, a b<0, \beta_{1}>\beta_{2}$, and $\psi>\beta_{2}$ or $\beta_{1}<0$ and $\psi<0$, then the implicit solution of equation (17) is

$$
\pm 2 \sqrt{-\frac{b}{3 a}}\left(\xi-\xi_{0}\right)=\frac{1}{\beta_{1}\left(\beta_{1}-\beta_{2}\right)} \ln \frac{\left[\sqrt{\beta_{1}\left(\psi-\beta_{2}\right)}-\sqrt{\psi\left(\beta_{1}-\beta_{2}\right)}\right]^{2}}{\left|\psi-\beta_{1}\right|}
$$

If $\varepsilon=1, a b<0, \beta_{1}>\beta_{2}$, and $\psi<0$ or $\beta_{1}<0$ and $\psi<\beta_{2}$, then the implicit solution of equation (17) is

$$
\pm 2 \sqrt{-\frac{b}{3 a}}\left(\xi-\xi_{0}\right)=\frac{1}{\beta_{1}\left(\beta_{1}-\beta_{2}\right)} \ln \frac{\left[\sqrt{-\beta_{1}\left(\psi-\beta_{2}\right)}-\sqrt{\psi\left(\beta_{2}-\beta_{1}\right)}\right]^{2}}{\left|\psi-\beta_{1}\right|} .
$$

If $\varepsilon=1, a b<0$, and $\beta_{2}>\beta_{1}>0$, then the implicit solution of equation (17) is

$$
\pm 2 \sqrt{-\frac{b}{3 a}}\left(\xi-\xi_{0}\right)=\frac{1}{\beta_{1}\left(\beta_{2}-\beta_{1}\right)} \arcsin \frac{\beta_{1}\left(\psi-\beta_{2}\right)+\psi\left(\beta_{1}-\beta_{2}\right)}{\beta_{2}\left|\psi-\beta_{1}\right|} .
$$

If $\varepsilon=-1, a b>0, \beta_{1}>\beta_{2}$, and $\psi>\beta_{2}$ or $\beta_{1}<0$ and $\psi<0$, then the implicit solution of equation (17) is

$$
\pm 2 \sqrt{\frac{b}{3 a}}\left(\xi-\xi_{0}\right)=\frac{1}{\beta_{1}\left(\beta_{2}-\beta_{1}\right)} \ln \frac{\left[\sqrt{\beta_{1}\left(-\psi+\beta_{2}\right)}-\sqrt{\psi\left(\beta_{1}-\beta_{2}\right)}\right]^{2}}{\left|\psi-\beta_{1}\right|}
$$

If $\varepsilon=-1, a b>0, \beta_{1}>\beta_{2}$, and $\psi<0$ or $\beta_{1}<0$ and $\psi<\beta_{2}$, then the implicit solution of equation (17) is

$$
\pm 2 \sqrt{\frac{b}{3 a}}\left(\xi-\xi_{0}\right)=\frac{1}{\beta_{1}\left(\beta_{2}-\beta_{1}\right)} \ln \frac{\left[\sqrt{-\beta_{1}\left(-\psi+\beta_{2}\right)}-\sqrt{\psi\left(\beta_{1}-\beta_{2}\right)}\right]^{2}}{\left|\psi-\beta_{1}\right|} .
$$

If $\varepsilon=-1, a b>0$, and $\beta_{2}>\beta_{1}>0$, then the implicit solution of equation (17) is

$$
\pm 2 \sqrt{\frac{b}{3 a}}\left(\xi-\xi_{0}\right)=\frac{1}{\beta_{1}\left(\beta_{1}-\beta_{2}\right)} \arcsin \frac{\beta_{1}\left(-\psi+\beta_{2}\right)+\psi\left(\beta_{2}-\beta_{1}\right)}{\beta_{2}\left|\psi-\beta_{1}\right|}
$$


Case 2.2. Assume that $\Delta=0$ and $D_{1}=0$. Denote $G(\psi)=\left(\psi-\beta_{1}\right)^{3}$, where $\beta_{1}$ is the real constant.
If $\varepsilon=1, \psi>\beta_{1}$, and $\psi>0$ or $\psi<\beta_{1}$ and $\psi<0$ then the explicit solution of equation (2) is

$$
u_{1}(t, x)= \pm\left[\frac{\beta_{1}}{-(b / 3 a) \beta_{1}^{2}\left(\left(x^{\alpha} / \alpha\right)-v\left(t^{\alpha} / \alpha\right)\right)-1}+\beta_{1}\right]^{1 / 2} e^{i\left(-k\left(x^{\alpha} / \alpha\right)+w\left(t^{\alpha} / \alpha\right)\right)}
$$

If $\varepsilon=-1, \psi>\beta_{1}$, and $\psi<0$ or $\psi<\beta_{1}$ and $\psi>0$, then the explicit solution of equation (2) is

$$
u_{2}(t, x)= \pm\left[\frac{\beta_{1}}{(b / 3 a) \beta_{1}^{2}\left(\left(x^{\alpha} / \alpha\right)-v\left(t^{\alpha} / \alpha\right)\right)-1}+\beta_{1}\right]^{1 / 2} e^{i\left(-k\left(x^{\alpha} / \alpha\right)+w\left(t^{\alpha} / \alpha\right)\right)}
$$

Case 2.3. Assume that $\Delta>0$ and $D_{1}<0$. Denote $G(\psi)=\left(\psi-\beta_{1}\right)\left(\psi-\beta_{2}\right)\left(\psi-\beta_{3}\right)$, where $0<\beta_{1}<$ $\beta_{2}<\beta_{3}$.

If $\varepsilon=1, \psi<0$, or $\psi>\beta_{3}$, then we make transformation $\psi=\left(\beta_{2} \beta_{3} \sin ^{2} \vartheta-\beta_{2} \beta_{3} / \beta_{3} \sin ^{2} \vartheta-\beta_{2}\right)$, and we obtain

$$
\pm \sqrt{-\frac{b}{3 a}}\left(\xi-\xi_{0}\right)=\frac{1}{\sqrt{\beta_{2}\left(\beta_{3}-\beta_{1}\right)}} \int \frac{\mathrm{d} \vartheta}{\sqrt{1-m_{1}^{2} \sin ^{2} \vartheta}}
$$

where $m_{1}^{2}=\left(\beta_{3}\left(\beta_{1}-\beta_{2}\right) / \beta_{2}\left(\beta_{1}-\beta_{3}\right)\right)$. Then, the explicit solution of equation (2) is

$$
u_{3}(t, x)= \pm\left[\frac{\beta_{2} \beta_{3} \operatorname{sn}^{2}\left(\sqrt{-(b / 3 a) \beta_{2}\left(\beta_{3}-\beta_{1}\right)}\left(\left(x^{\alpha} / \alpha\right)-v\left(t^{\alpha} / \alpha\right)\right), m_{1}\right)-\beta_{2} \beta_{3}}{\beta_{3} \operatorname{sn}^{2}\left(\sqrt{-(b / 3 a) \beta_{2}\left(\beta_{3}-\beta_{1}\right)}\left(\left(x^{\alpha} / \alpha\right)-v\left(t^{\alpha} / \alpha\right)\right), m_{1}\right)-\beta_{2}}\right]^{1 / 2} e^{i\left(-k\left(x^{\alpha} / \alpha\right)+w\left(t^{\alpha} / \alpha\right)\right)}
$$

If $\varepsilon=1$ and $\beta_{1}<\psi<\beta_{2}$, then we make transformation $\psi=\left(-\beta_{1} \beta_{2} /\left(\beta_{2}-\beta_{1}\right) \sin ^{2} \vartheta-\beta_{2}\right)$. Similarly, the explicit solution of equation (2) is

$$
u_{4}(t, x)= \pm\left[\frac{-\beta_{1} \beta_{2}}{\left(\beta_{2}-\beta_{1}\right) s n^{2}\left(\sqrt{-(b / 3 a) \beta_{2}\left(\beta_{3}-\beta_{1}\right)}\left(\left(x^{\alpha} / \alpha\right)-v\left(t^{\alpha} / \alpha\right)\right), m_{1}\right)-\beta_{2}}\right]^{1 / 2} e^{i\left(-k\left(x^{\alpha} / \alpha\right)+w\left(t^{\alpha} / \alpha\right)\right)}
$$

If $\varepsilon=-1$ and $\beta_{2}<\psi<\beta_{3}$, then we make transformation $\psi=\left(\beta_{1}\left(\beta_{3}-\beta_{2}\right) \sin ^{2} \vartheta-\beta_{2}\left(\beta_{3}-\beta_{1}\right) /\left(\beta_{3}-\right.\right.$ $\left.\left.\beta_{2}\right) \sin ^{2} \vartheta-\left(\beta_{3}-\beta_{1}\right)\right)$, and the explicit solution of equation (2) is

$$
u_{5}(t, x)= \pm\left[\frac{\beta_{1}\left(\beta_{3}-\beta_{2}\right) s n^{2}\left(\sqrt{-(b / 3 a) \beta_{2}\left(\beta_{3}-\beta_{1}\right)}\left(\left(x^{\alpha} / \alpha\right)-v\left(t^{\alpha} / \alpha\right)\right), m_{2}\right)-\beta_{2}\left(\beta_{3}-\beta_{1}\right)}{\left(\beta_{3}-\beta_{2}\right) s n^{2}\left(\sqrt{-(b / 3 a) \beta_{2}\left(\beta_{3}-\beta_{1}\right)}\left(\left(x^{\alpha} / \alpha\right)-v\left(t^{\alpha} / \alpha\right)\right), m_{2}\right)-\left(\beta_{3}-\beta_{1}\right)}\right]^{1 / 2} e^{i\left(-k\left(x^{\alpha} / \alpha\right)+w\left(t^{\alpha} / \alpha\right)\right)}
$$

where $m_{2}^{2}=\left(\beta_{1}\left(\beta_{2}-\beta_{3}\right) / \beta_{2}\left(\beta_{1}-\beta_{3}\right)\right)$. 
If $\varepsilon=-1$ and $0<\psi<\beta_{1}$, then we make transformation $\quad \psi=\left(\beta_{1} \beta_{3} \sin ^{2} \vartheta / \beta_{1} \sin ^{2} \vartheta-\left(\beta_{1}-\beta_{3}\right)\right)$.

Similarly, the explicit solution of equation (2) is

$$
u_{6}(t, x)= \pm\left[\frac{\beta_{1} \beta_{2} s n^{2}\left(\left(\left(x^{\alpha} / \alpha\right)-v\left(t^{\alpha} / \alpha\right) / 2\right) \sqrt{(b / 3 a) \beta_{2}\left(\beta_{3}-\beta_{1}\right)}, m_{2}\right)}{\beta_{1} s n^{2}\left(\left(\left(x^{\alpha} / \alpha\right)-v\left(t^{\alpha} / \alpha\right) / 2\right) \sqrt{(b / 3 a) \beta_{2}\left(\beta_{3}-\beta_{1}\right)}, m_{2}\right)-\left(\beta_{1}-\beta_{3}\right)}\right]^{1 / 2} e^{i\left(-k\left(x^{\alpha} / \alpha\right)+w\left(t^{\alpha} / \alpha\right)\right)}
$$

Case 2.4. Assume that $\Delta<0$. Denote $G(\psi)=\left(\psi-\beta_{1}\right)\left[\left(\psi-\beta_{2}\right)^{2}+\gamma\right]$, where $\beta_{1}, \beta_{2}, \gamma>0$ are real constants. Then, we make transformation $\psi=\left(d_{1} \cos \vartheta+d_{2} / d_{3} \cos \vartheta+d_{4}\right)$, where $d_{1}=\left(\beta_{1} /\right.$ 2) $\left(d_{3}-d_{4}\right), d_{2}=-\left(\beta_{1} / 2\right)\left(d_{3}-d_{4}\right), d_{3}=\beta_{1}-\beta_{2}-$ $(\gamma / m), d_{4}=\beta_{1}-\beta_{2}-\gamma m, m=D \pm \sqrt{D^{2}+1}$, and $D=\left(\gamma^{2}-\beta_{2}\left(\beta_{1}-\beta_{2}\right) / \beta_{2} \gamma\right)$.

If $\varepsilon=1$ and $m=D-\sqrt{D^{2}+1}$, then the explicit solution of equation (2) is

$$
u_{7}(t, x)= \pm\left[\frac{d_{1} c n\left(\left(\left(x^{\alpha}-v t^{\alpha} / \alpha\right) \sqrt{2(b / 3 a) \gamma m \beta_{1}} / m m_{3}\right), m_{3}\right)+d_{2}}{d_{3} c n\left(\left(\left(x^{\alpha}-v t^{\alpha} / \alpha\right) \sqrt{2(b / 3 a) \gamma m \beta_{1}} / m m_{3}\right), m_{3}\right)+d_{4}}\right]^{1 / 2} e^{i\left(-k\left(x^{\alpha} / \alpha\right)+w\left(t^{\alpha} / \alpha\right)\right)}
$$

If $\varepsilon=-1$ and $m=D+\sqrt{D^{2}+1}$, then the explicit solution of equation (2) is

$$
u_{8}(t, x)= \pm\left[\frac{d_{1} c n\left(\left(\left(x^{\alpha}-v t^{\alpha} / \alpha\right) \sqrt{-2(b / 3 a) \gamma m \beta_{1}} / m m_{3}\right), m_{3}\right)+d_{2}}{d_{3} c n\left(\left(\left(x^{\alpha}-v t^{\alpha} / \alpha\right) \sqrt{-2(b / 3 a) \gamma m \beta_{1}} / m m_{3}\right), m_{3}\right)+d_{4}}\right]^{1 / 2} e^{i\left(-k\left(x^{\alpha} / \alpha\right)+w\left(t^{\alpha} / \alpha\right)\right)}
$$

where $m_{3}^{2}=\left(1 / 1+m^{2}\right)$.

\section{Conclusion}

In the paper, the traveling wave solution of the fractional pGI equation is studied by using the complete discrimination system method. Firstly, we reduce equation (2) to the nonlinear ordinary differential equation by fractional traveling wave transformation. Then, the complete discrimination system is used to construct the classification of all single traveling wave solutions of the fractional pGI equation. Among them, the implicit solutions have not been reported in other literatures. Compared with the existing literature [15-17], our solutions are more abundant, including rational function solutions, Jacobian function solutions, and implicit solutions. It is worth mentioning that the complete discrimination system method is an effective and powerful tool for constructing the traveling wave solution fractional pGI equation. Moreover, the method can be employed to construct the traveling wave solution of general FPDE.

\section{Data Availability}

No data were used to support this study.

\section{Conflicts of Interest}

The authors declare that they have no conflicts of interest.

\section{Acknowledgments}

This work was supported by Institutions of Higher Education of Sichuan Province under grant no. MSSB-2021-13.

\section{References}

[1] M. M. A. Khater, S. Anwar, K. U. Tariq, and M. S. Mohamed, "Some optical soliton solutions to the perturbed nonlinear Schrödinger equation by modified khater method," AIP Advances, vol. 11, Article ID 25130, 2021.

[2] Y. Xie, L. Li, and Y. Kang, "New solitons and conditional stability to the high dispersive nonlinear Schrödinger equation with parabolic law nonlinearity," Nonlinear Dynamics, vol. 103, pp. 1011-1021, 2021.

[3] T. Han, L. Zhao, and X. Zhang, "Bifurcation and new exact traveling wave solutions to time-space coupled fractional nonlinear Schrödinger equation," Physics Letters A, vol. 395, Article ID 127217, 2021.

[4] Y. Xie, Z. Yang, and L. Li, "New exact solutions to the high dispersive cubic-quintic nonlinear Schrödinger equation," Physics Letters A, vol. 382, no. 36, pp. 2506-2514, 2018. 
[5] L. Du, Y. Sun, and D. Wu, "Bifurcations and solutions for the generalized nonlinear Schrödinger equation," Physics Letters A, vol. 383, no. 36, Article ID 126028, 2019.

[6] B. He and Q. Meng, "Bifurcations and new exact travelling wave solutions for the Gerdjikov-Ivanov equation," Communications in Nonlinear Science and Numerical Simulation, vol. 15, no. 7, pp. 1783-1790, 2010.

[7] A. Biswas, Y. Yildirim, E. Yasar et al., "Solitons for perturbed Gerdjikov-Ivanov equation in optical fibers and PCF by extended Kudryashov's," Optical and Quantum Electronics, vol. 50, p. 149, 2018.

[8] S. Arshed, "Two reliable techniques for the soliton solutions of perturbed Gerdjikov-Ivanov equation," Optik, vol. 164, pp. 93-99, 2018.

[9] S. Arshed, A. Biswas, M. Abdelaty, Q. Zhou, S. Moshokoa, and M. R. Belić, "Optical soliton perturbation for GerdjikovIvanov equation invia two analytical techniques," Chinese Journal of Physics, vol. 56, no. 6, pp. 2879-2886, 2018.

[10] A. Muniyappan, P. Monisha, E. K. Priya, and V. Nivetha, "Generation of wing-shaped dark soliton for perturbed Gerdjikov-Ivanov equation in optical fibre," Optik, vol. 230, Article ID 166328, 2021

[11] K. Hosseini, M. Mirzazadeh, M. Ilie, and S. Radmehr, "Dynamics of optical solitons in the perturbed Gerdjikov-Ivanov equation," Optik, vol. 206, Article ID 164350, 2020.

[12] R. Khalil, M. Al Horani, A. Yousef, and M. Sababheh, "A new definition of fractional derivative," Journal of Computational and Applied Mathematics, vol. 264, pp. 65-70, 2014.

[13] L. Zhao, T. Han, and C. Huang, "Bifurcation and new exact traveling wave solutions for time-space fractional Phi-4 equation," AIP Advances, vol. 10, no. 11, Article ID 115113, 2020.

[14] L. Zhao and T. Han, "Bifurcation and exact solutions for the $(2+1)$-dimensional conformable time-fractional Zoomeron equation," Advances in Difference Equations, vol. 2020, p. 656, 2020.

[15] C. Li, G. Li, and L. Chen, "Fractional optical solitons of the space-time perturbed fractional Gerdjikov-Ivanov equation," Optik, vol. 224, Article ID 165638, 2020.

[16] B. Ghanbari and B. Dumitru, "New optical solutions of the fractional Gerdjikov-Ivanov equation with conformable derivative," Frontiers in Physics, vol. 8, p. 167, 2020.

[17] A. Zulfiqar and J. Ahmad, "New optical solutions of conformable fractional perturbed Gerdjikov-Ivanov equation in mathematical nonlinear optics," Results in Physics, vol. 21, Article ID 103825, 2021.

[18] L. Li, Z. Yang, and Y. Xie, "New exact solutions for a generalized KdV equation," Nonlinear Dynamics, vol. 95, pp. 215-219, 2018. 